\title{
Comunidades académicas bioaprendientes: Emergencias autoorganizativas en el contexto de la educación superior ${ }^{7}$
}

\author{
Bioaprendientes academic communities: emergency self-organizing in the context of higher education
}

Recibido 28 febrero 2014 • Aceptado 13 junio 2014 • Corregido 26 junio 2014

\author{
Luz Mery Chaverra Rodríguez ${ }^{2}$ \\ Universidad Católica de Manizales
}

Manizales, Colombia

Ichaverra@ucm.edu.co; merycharo1@hotmail.com

\author{
Faber Andrés Alzate Ortiz ${ }^{3}$ \\ Universidad Católica de Manizales \\ Manizales, Colombia \\ falzate@ucm.edu.co; faberalzate@hotmail.com
}

\begin{abstract}
Resumen. El presente artículo corresponde a los avances conceptuales de la investigación "Comunidades académicas bioaprendientes: emergencias autoorganizativas en el contexto de la educación superior" que, en la actualidad, se gesta como tesis doctoral en la Universidad de La Salle, Costa Rica. Este proyecto de investigación asume el bioaprendizaje como alternativa para el campo de la gestión en las instituciones de educación superior; propuesta que pretende estimular la construcción colectiva de comunidades académicas capaces de funcionar como sistemas vivos, autoorganizados, dinámicos y contextualizados con oportunidades y necesidades internas y externas. Esta reflexión teórica centra su atención en aquellas tendencias, modelos y prácticas de la gestión educativa que merecen ser reconfiguradas/resignificadas con el fin de colocarlas a tono con la época, la cual demanda otras maneras de convivir, habitar, trabajar y, además, con otras formas de relacionamiento donde participan los otros y lo otro.
\end{abstract}

Palabras claves. Bioaprendizaje; comunidades académicas; organizaciones vivas; gestión humanizante.

1 EL presente artículo es producto de las revisiones teóricas que se han adelantado respecto a la categoría de bioaprendizaje, en el marco de la tesis doctoral denominada "Comunidades académicas bioaprendientes: emergencias autoorganizativas en el contexto de la educación superior", inscrita en el programa de Doctorado en Educación con énfasis en Mediación Pedagógica de la Universidad de La Salle, Costa Rica.

2 Decana de la Facultad de Educación de la Universidad Católica de Manizales, Colombia. Estudiante de Doctorado en Educación con énfasis en Mediación Pedagógica, Universidad de La Salle, Costa Rica. Magister en Docencia Superior con énfasis en Educación de Adultos; Especialista en Educación Personalizada y Licenciada en Filosofía y Ciencias Religiosas. Investigadora del Grupo de Investigación, Educación y Formación de Educadores.

3 Director de las especializaciones en Educación de la Universidad Católica de Manizales, Colombia. Estudiante de Doctorado en Educación con énfasis en Mediación Pedagógica, Universidad de La Salle, Costa Rica. Magíster en Educación y Licenciado en Gestión Educativa. Investigador del Grupo de Investigación, Educación y Formación de Educadores.

Licencia Creative Commons

Atribución-No-Comercial SinDerivadas

3.0 Costa Rica.

Luz Mery Chaverra Rodríguez y Faber Andrés Alzate Ortiz 
Abstract. This article corresponds to the conceptual advances of research " bioaprendientes academic communities : emergency self-organizing in the context of higher education ", which currently is brewing as a doctoral thesis at the University of the Salle de Costa Rica, research project in which learning takes bio - alternative to the field of management in higher education institutions. This is a proposal that aims to stimulate the collective construction of academic communities capable of functioning as living systems, self-organized, dynamic and contextualized with the opportunities and internal and external needs. This theoretical reflection focuses on the trends, patterns and practices of educational management that deserve re-configuradas/re-significadas order to place them in tune with the times, which demands, other ways to live, dwell , work and also to other forms of relationship which involved the other and that.

Keywords. Bio-learning; academic communities; living organizations; humanizing management

\section{INTRODUCCIÓN}

“Hablar hoy día de nichos vitales -y no hay vida sin ellos- significa hablar de ecologías cognitivas, de

(Assman, 2002, p. 23)

En esta reflexión teórica se plantean algunas consideraciones conceptuales y prácticas alrededor de la gestión educativa que pueden ser de gran utilidad para quienes se desempeñan como directivos y docentes en instituciones de educación superior. Desempeño que está determinado no solo por la capacidad creadora de las personas, sino también por las formas en que emergen las relaciones interpersonales $y$, con ello, las maneras de gestionar el talento humano y las relaciones de poder entre los líderes y sus equipos (docentes y directivos universitarios).

Como se observará más adelante, uno de los asuntos importantes dentro de los procesos de resignificación y mejoramiento institucional, desde la perspectiva del desempeño, está anclado en la promoción e inclusión de aspectos como el respeto por la diferencia, el trabajo en equipo, la ética profesional, el empoderamiento y la confianza recíproca en la cultura/clima organizacional. Estos exigen abordar otras formas de trabajar, convivir y gestionar el conocimiento, de manera que se construya valor organizacional duradero, pertinente y altamente calificado en todos los niveles y colectivos humanos (Hellriegel \& Slocum, 2009; Lessem, 1992).

En este orden de ideas, con la propuesta de generar comunidades académicas bioaprendientes, se pretende movilizar a los equipos y a sus líderes hacia procesos de autorregulación y autoorganización que potencien un quehacer transformador, democrático e innovador, a partir del cual sea posible consolidar una organización que se geste como un sistema vivo, que supere los actuales modelos económicos donde suele valorarse más el consumismo de

Licencia Creative Commons 
Rev. Gestión de la Educación, Vol. 4, N² 2, [119-129], ISSN: 2215-2288, julio-diciembre, 2014

DOI: http://dx.doi.org/10.15517/rge.v4i2.15147

URL: http://revistas.ucr.ac.cr/index.php/gestedu

bienes materiales y trascienda hacia los valores de una convivencia sociocultural respetuosa de las diferencias, en el marco de la promoción de los valores esenciales para el bienestar humano en sociedades justas y equitativas.

La pretensión ética yacadémica de esteartículo es analizar los diferentes vacíos teórico-prácticos que, a criterio de sus autores, es posible observar en los procesos de gestión de las instituciones de educación superior, desde una perspectiva crítico-hermenéutica y proponer un camino posible de reflexión y resignificación basado en un pensar reflexivo, abierto, sensible y complejo.

\section{REFLEXIONES TEÓRICO-CONCEPTUALES}

\section{Hacia una cultura de la autoorganización y el aprendizaje colaborativo}

Fomentar procesos de autoorganización en los diferentes escenarios académicos y directivos en las instituciones deeducación superior sugiere, inicialmente, identificar la capacidad que tienen estas de constituirse y reconocerse como un sistema vivo, en perspectiva de lo que Maturana (2007) define como dinámica autopoiética. Esta tiene una condición ineludible en el desarrollo de los sistemas y es la posibilidad de re-crear-se a sí mismas sin importar su naturaleza jurídica, ya se pública o privada. En este sentido, las comunidades educativas podrán generar procesos de mejoramiento y autogestión desde dinámicas que den cuenta de un pensar-actuar organizador y creador que, en palabras de Bohm (1998), serían sistemas/comunidades en "flujo continuo" (p. 2), con la participación de los diferentes actores sociales.

Lo anterior, permite comprender que cualquier estructura organizacional, aunque tenga cierto nivel de autonomía y estabilidad, siempre requiere acciones colectivas para gestionar-se y movilizar-se hacia niveles de innovación y transformación a partir de los cuales sea posible romper con aquellos dogmas, paradigmas, ideologías institucionalizados e instituyentes que limitan, significativamente, toda posibilidad de renovación y crecimiento.

Esta realidad no es ajena a lo que en la actualidad viven algunas instituciones de educación superior, tanto públicas como privadas, las cuales muestran signos de estar orientadas por una cultura de la conservación, la linealidad, la repetición y la programación excesiva que funcionan, de alguna manera, como agentes restrictivos que sutilmente pareciesen condicionar/supeditar la acción creadora, innovadora y transformadora de sus equipos humanos (Puchol, 2005; Menchén, 2009).

Superar las dinámicas inflexibles/dominantes/heterónomas en las que se encuentran este grupo de instituciones significa, en palabras de Eisler (1998a), "desmitificar los estereotipos dominadores e inspirarnos a escribir nuestros propios guiones de vida" (p. 240); lo que significa apostar por la recuperación del horizonte organizacional como sujetos en común-unidad que, en palabras de Morín (2003), es un llamado a ser sujetos planetarios, capaces de establecer relaciones armoniosas, equilibradas y dinámicas, posibilitadoras de prácticas del diálogo, en

Licencia Creative Commons 
apertura y sincrónicas con las necesidades y oportunidades del entorno, desde una visión integradora y cooperativa, totalmente contrarias a las impuestas por las políticas neoliberales del actual contexto socioeconómico (Bohm, 1996).

Las instituciones educativas están invitadas a hacer ejercicios reflexivos alrededor de aquellas normas/preceptos/estatutos que orientan su vida y obra socioeducativa, que han sido las que dan cuenta de su cultura organizacional, la cual, históricamente, se va tejiendo según Eisler (1998a), "a partir de las historias que se han contado de generación en generación y con las que se han creado inconscientemente los propios guiones de vida institucional" (p. 223); tejido organizacional que debe despertar una voluntad colectiva lo que denomina Legendre, citado por Morín (2006), como "autoservicio normativo" (p. 30).

Lo anterior, con la finalidad de reconocer aquellos lineamientos y directrices que orientan la vida institucional y que han sido pensados/diseñados, algunos de ellos, para imponer, discriminar, segregar e invisibilizar al otro y con ello, limitar su carga simbólica de potencial creador hasta el punto de dejarlo muy lejos de toda posibilidad de crecimiento y realización personal y profesional. En este sentido, se retomarían lineamientos con los que se puede apostar por la promoción de relaciones de poder basados en criterios de igualdad, equidad, democracia, responsabilidad y respeto que propicien el reconocimiento de la dignidad de cada persona, indistinta sea su condición laboral, de género, etarias, social, económica, étnica y profesional al interior de la organización.

Se observa hasta aquí, lo posible y necesario que es explorar la posibilidad de concebir las instituciones de educación superior como organizaciones vivas, autorreguladas, con capacidad para autogestionar-se y entrar en contacto vital con el entorno que las rodea, creando tramas/ urdimbres bioaprendientes, en función de las dimensiones epistemológicas y ontológicas del conocimiento que son producto emergente de la investigación, la docencia y la extensión que, social y científicamente, las caracteriza.

La institución educativa, como organización que se gestiona desde procesos de bioaprendizaje, supone importantes movilizaciones/cambios en aquellos imaginarios radicales que se apoyan en paradigmas administrativos verticales y burocráticos basados en el control, el autoritarismo y el liderazgo transaccional, promoviéndose más bien la consolidación de organizaciones que fortalecen una perspectiva cultural del aprendizaje a partir de la búsqueda permanente de conocimiento, resignificación de las prácticas y el trabajo colaborativo, como señala Bolívar (2007), "las organizaciones aprenden cuando tienen competencias para ser sensibles a las demandas del entorno, pueden adquirir conocimientos y emplearlos para incrementar la efectividad de sus respuestas, y una vez detectados errores son capaces de responder creativamente" (p. 150).

Licencia Creative Commons 


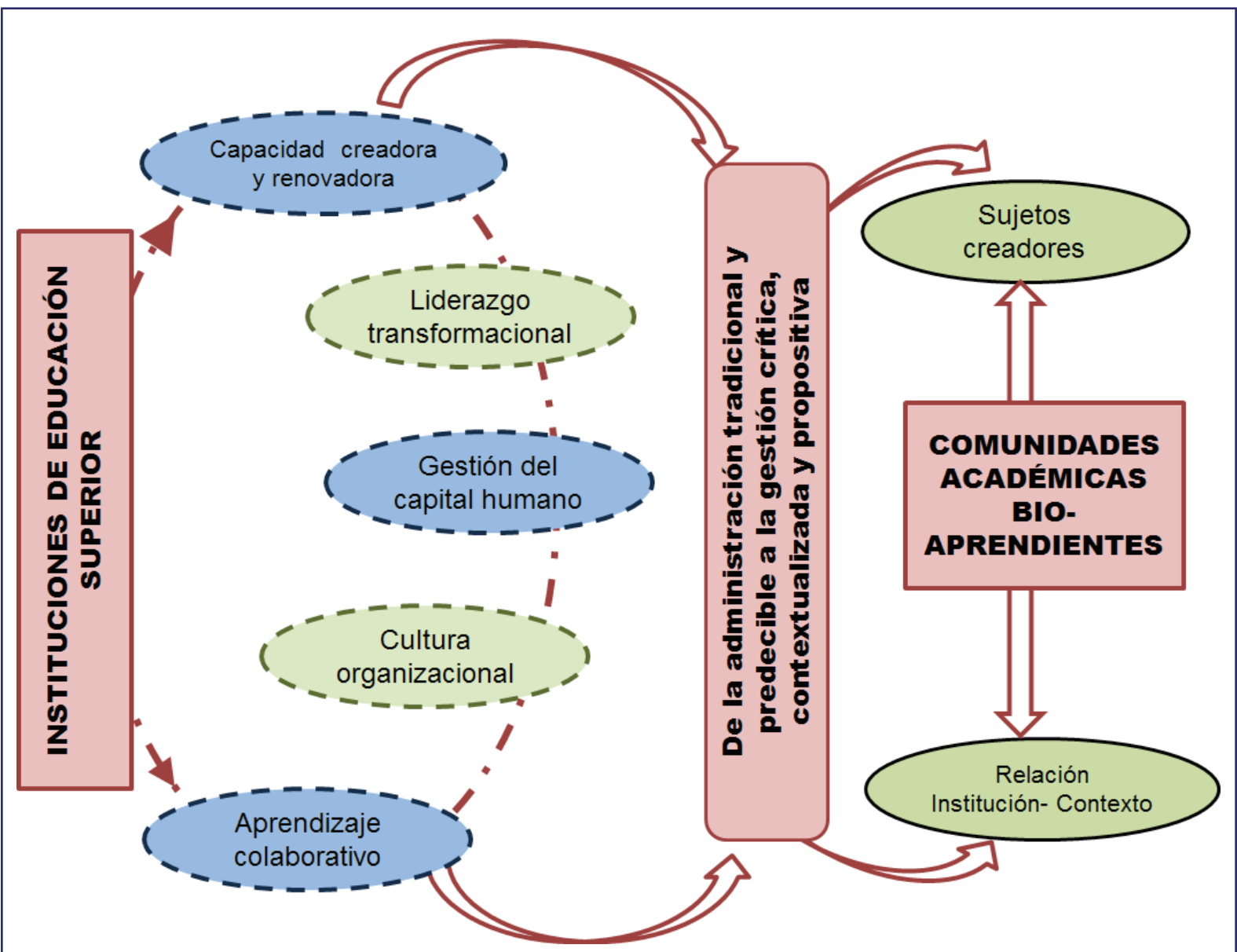

Figura 1. Tránsitos de la gestión desde el bioaprendizaje. Elaboración propia.

En la lógica del bioaprendizaj, como lo plantearía Asmman (2002), “los seres vivos son seres que consiguen mantener de forma flexible y adaptativa la dinámica de seguir aprendiendo" ( $p$. 23) y esto, en el contexto de las instituciones educativas, evoca un llamado ético y transformador para la alta dirección, si se tiene en cuenta, además de todo lo hasta aquí planteado, que a cada miembro de la organización es importante permitirle experimentar-se e implicar-se en los procesos como un "Yo creador" (Swimme, 1998, p. 35), con capacidad de desarrollar actividades que, en sintonía con su potencial humano y perfil profesional, le aporten valor a la organización.

Finalmente, como lo propone Calvo (2009), sería abandonar las autopistas y avenidas de lo establecido -lo seguro, lo que ya está previsto- para transitar por el camino de la incertidumbre, lo inimaginable, lo incierto, lo imprevisto, manteniendo viva conciencia de la responsabilidad 
de cada miembro de la institución de trazar nuevos senderos para "pasar del predominio de las historias construidas por otros al valor de las historias que se construyen" (Novo, 2007, p. 39).

\section{De una administración de las normas y el control, a una gestión humanizante y colaborativa}

Las instituciones de educación superior son un reflejo posible de una sociedad en miniatura, en las que emergen las lógicas de poder basadas en concepciones verticales, burocráticas o deterministas que colocan a las personas en condición de sometimiento, limitando su potencial creador o lógicas de poder, basadas en el liderazgo transformador que convoca y democratiza las decisiones y el conocimiento, potencia y estimula al ser humano y hace del aprendizaje, una oportunidad de desarrollo individual y colectivo (Brenes, 2003).

Salk, citado por Eisler (1998a), afirma que "nuestra necesidad más urgente está en suministrar a aquel maravilloso instrumento, la mente humana, los medios de imaginar $y$, consecuentemente, crear un mundo mejor" (p. 128). Significa que, al favorecer un tejido de relaciones y dependencias recíprocas y esenciales, se le permite a la organización/comunidad educativa fortalecer su dinamismo biocreador. De esta manera, se requiere movilizar, en la institución, la capacidad de desprendimiento de lo estático, lo preestablecido, lo rutinario, porque las estructuras organizacionales no deben asumir posiciones absolutas e inflexibles, sino abrirse y afrontar las nuevas emergencias humanas, políticas, culturales, socioeducativas, científicas y tecnológicas, que están llevando a nuevas creaciones, nuevas realidades, nuevas hermenéuticas del mundo de la vida y del trabajo, nuevas maneras de gestionar/gerenciar y hasta nuevas formas de habitar-nos en la sociedad (Pérez, 1991; Casado, 2001).

En palabras de Eisler (1998b), "es propiciar el despertar de la conciencia individual y colectiva de que tenemos opciones y podemos hacer cambios y que estos son esenciales" ( $p$. 224), manteniendo la dinámica del aprendizaje organizacional como un proceso vital, abierto, complejo y constante en todos los actores y niveles del sistema institucional, para lo cual, además, "es indispensable cambiar las ideas, de modo que vivamos en armonía"(Najmanovich, 2008, p. 52).

Antes que mantener hábitos de pensamiento, opiniones y experiencias que giran una y otra vez para mantener a las personas y a los equipos de trabajo en el mismo lugar y en las mismas dinámicas, denominado por Briggs y Peat (1999) como"rizos de retroalimentación negativos" ( $p$. 37), es necesario reconocer la posibilidad que hay en cada uno de los actores de la organización para propiciar desde el diálogo constructivo, interdependiente y horizontal, procesos de rede-construcción de prácticas académicas y directivas que impulsen su mejoramiento continuo (Cummings y Worley, 2007).

Con el bioaprendizaje es posible concebir una institución de educación superior que vitaliza su quehacer desde la experiencia enriquecedora y sensible del diálogo como encuentro directo

Licencia Creative Commons 
que posibilita la armonía, el cooperativismo, la corresponsabilidad y el encanto de vivir, de estar y trabajar juntos. Lo anterior, propicia en los directivos acciones encaminadas a estimular y potenciar el desarrollo de los colaboradores/miembros de sus equipos, inicialmente desde expresiones como:"te creo", "puedes", "asume el riesgo", "disfruta", "resuelve" "confía", "es posible", etc.

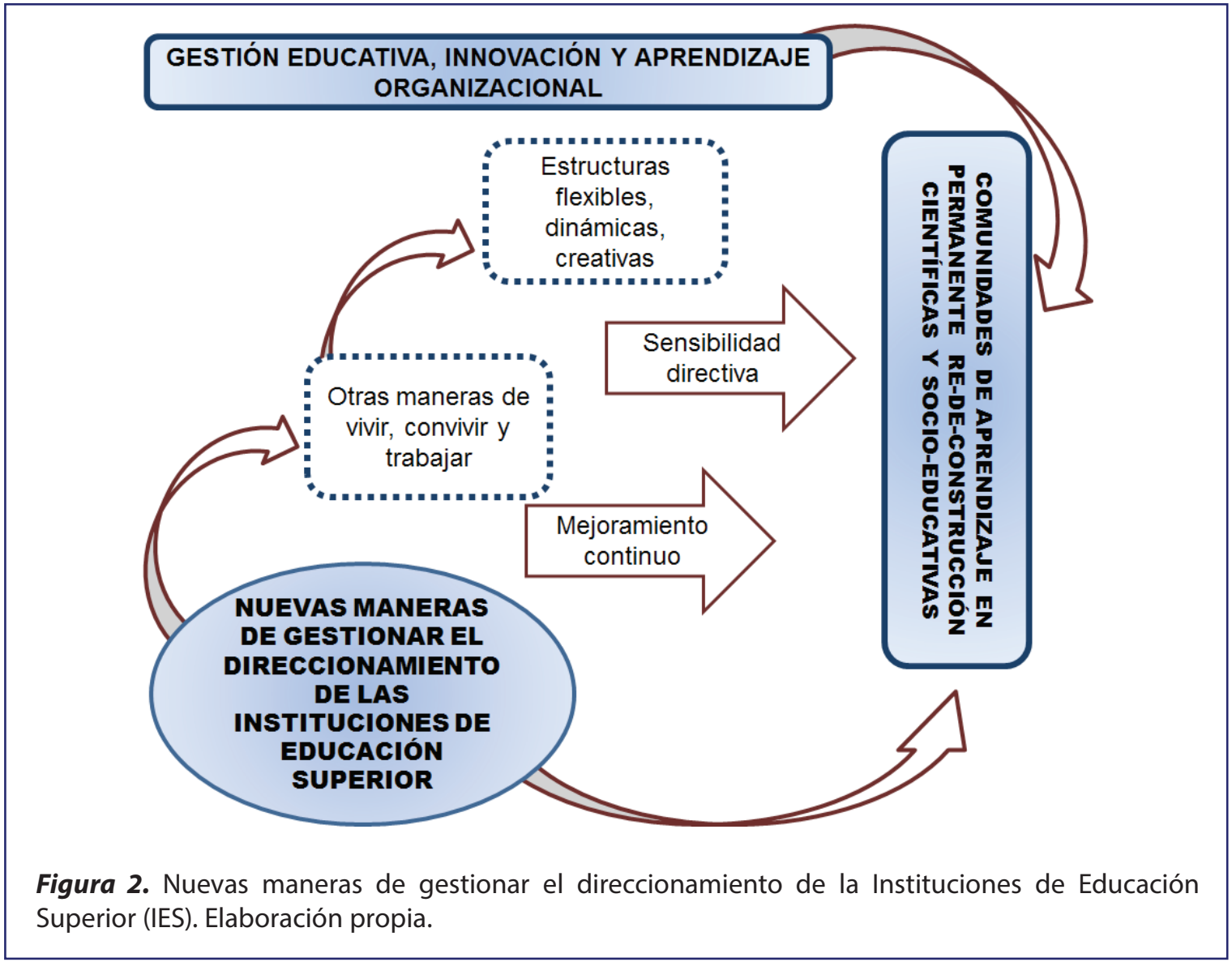

\section{Lógicas del bioaprendizaje en el mejoramiento de las comunidades académicas}

Como se ha insistido hasta aquí, las comunidades académicas bioaprendientes que se gestan en el contexto de la educación superior, son aquellas capaces de impulsar sus desarrollos científicos y socioeducativos a partir de procesos de dirección horizontales, dialogantes, interdependientes, sensibles con el otro y lo otro, críticos, flexibles e innovadores; todo en el marco de un ambiente de trabajo/clima organizacional que promueva una adecuada y sana convivencia, a partir de la cual, desde el compartir unos con otros, sea posible socializar/ reconocer y potenciar adecuadamente los intereses, inquietudes, problemas, expectativas, 
oportunidades de todas y todos. Asuntos/aspectos que tejen no solo la identidad institucional, sino otros conocimientos vitales en la construcción de valor para la institución (Estebaranz, 2000).

Dado el carácter social del ser humano, la convivencia se constituye, según lo expresa Maturana (2007), en el medio natural en el que"las retroacciones e interacciones que construyen al ser humano en la sociedad para compartir, transformar y generar conocimiento, hace que esta evolucione, que él evolucione, que sea un sistema adaptativo, complejo y evolutivo" ( $p$. 4), admitiéndose en este sentido, para el caso de las comunidades bioaprendientes, que es necesario que a todos los miembros implicados en las decisiones y obra de la institución, se les permita participar activamente desde la naturaleza misma de su esencia personal y profesional. Esto se expresa en el contexto organizacional, de manera singular y subjetiva, desde acciones como: soñar, amar, disentir, debatir, dudar, desconfiar, creer, imaginar, compartir, indagar, etc., las cuales son tan importantes como los criterios técnicos o teóricos en los que se basaría un directivo para tomar determinadas decisiones.

En este sentido, es necesario propiciar un ambiente de trabajo donde sea privilegiada una sana y constructiva convivencia. Así, más que el autoritarismo, las decisiones/comportamientos radicales, la opresión y el sometimiento, prevalecen acciones directivas que promueven el trabajo colegiado, la coinspiración académica, el intercambio de saberes y el reconocimiento de todos y cada uno de los actores desde su experiencia de vida, bagaje profesional y modos de conocer y hacer. De esta manera, pueden generar experiencias laborales renovadas y significativas que favorezcan positivamente a la organización porque, de acuerdo con Maturana (1992), "o se vive en el bienestar estético de una convivencia armónica, o en el sufrimiento de la exigencia negadora continua" (p. 18).

En términos de Sheldrake (1994), se genera lo que se podría denominar el efecto resonancia, entendido como la implicancia de todos los equipos de trabajo en la generación de otras realidades, nuevos conocimientos, nuevas maneras de innovar y crear valor y, desde allí, posibilitar transformaciones en la vida de las personas y de la organización. Asuntos que, en el contexto del bioaprendizaje y de la gestión de comunidades académicas de alto nivel, delimitan al menos cuatro recomendaciones claves para los directivos:

1. Promover un liderazgo transformador que potencie la condición humana desde el reconocimiento de las habilidades y saberes que posean las personas.

2. Generar condiciones laborales para que las personas perciban la organización como un espacio vital, en el que es posible desplegar su potencial físico, intelectual y espiritual.

3. Promover procesos permanentes de aprendizaje organizacional dinámicos, flexibles, abiertos, dialógicos y constructivos que transformen la manera de ser y el quehacer en la institución.

Licencia Creative Commons 
Rev. Gestión de la Educación, Vol. 4, N² 2, [119-129], ISSN: 2215-2288, julio-diciembre, 2014

DOI: http://dx.doi.org/10.15517/rge.v4i2.15147

URL: http://revistas.ucr.ac.cr/index.php/gestedu

4. Desarrollar estrategias directivas que posibiliten el trabajo colaborativo, la autorregulación, la humanización de la gestión y la innovación permanente.

En este orden de ideas, se esperaría que en las instituciones de educación superior emerja una gestión directiva revitalizada que favorezca procesos de mejoramiento y desarrollo organizacional a pequeña y gran escala, generándose procesos de bioaprendizaje que estén más allá de discursos o intenciones pasajeras, y que se conviertan en parte indeleble de la cultura organizacional.

\section{CONCLUSIONES}

En relación con el marco reflexivo expuesto, se establecen las siguientes conclusiones:

- Pasar de una administración de las normas y el control a una gestión humanizante y colaborativa, representa un compromiso institucional por consolidar modelos de gestión que potencien la persona y el aprendizaje, a partir del reconocimiento y la valoración de sus capacidades individuales y colectivas. En este sentido, las estrategias de gestión se situarán en contextos de realidad que posibiliten la participación democrática, emancipadora y colaborativa de todos y todas sus integrantes. Las instituciones de educación superior serán, de esta manera, espacios vitales de despliegue en humanidad, en los cuales se privilegia una gestión integral del conocimiento.

- Construir una cultura organizacional basada en la autoorganización y el aprendizaje colaborativo es posible, en la medida en que la gestión educativa que desarrollan los directivos de las instituciones de educación superior esté basada en la promoción permanente de procesos de bioaprendizaje, de tal manera que se promueva el desarrollo creativo y sensible de las personas y sus equipos de trabajo; emergiendo a la vez, de manera natural, un liderazgo compartido y transformador capaz de hacer de la organización un escenario vital a partir de la innovación, la investigación y el desarrollo.

- En función de las lógicas del bioaprendizaje en el mejoramiento de las comunidades académicas, es necesario destacar que las comunidades académicas bioaprendientes son equipos humanos que están en capacidad de desarrollar exitosamente los objetivos y metas organizacionales basándose en la autoorganización, autogestión y autorregulación de sus capacidades y responsabilidades. Esto exige a sus líderes, propiciar condiciones laborales que favorezcan el trabajo en equipo, las relaciones interpersonales armoniosas, el sentido de pertenencia, el desarrollo de la creatividad, la optimización del tiempo y los recursos y otro sinnúmero de estrategias de gestión humanizante que puedan servir en la generación valor a la organización.

Licencia Creative Common 


\section{Referencias}

Assman, H. (2002). Placer y ternura en la educación. Madrid: Narcea S.A. Ediciones.

Bohm, D. (1996). Sobre el diálogo. Barcelona: Editorial Kairós.

Bohm, D. (1998). La totalidad y el orden implicado. Barcelona: Editorial Kairós.

Bolivar, A. (2007). Los centros educativos como organizaciones que aprenden. Promesas y realidades. Madrid: Editorial La Muralla.

Brenes, B. L. (2003). Dirección estratégica para organizaciones inteligentes. San José, Costa Rica: Editorial Universidad Estatal a Distancia.

Briggs, J. y Peat, D. (1999). Las siete leyes del caos. Barcelona: Editorial Grijalbo.

Calvo, C. (2009). Los procesos educativos y la emergencia de complejidades caóticas y autoorganizadas. Revista Acción Pedagógica, 18(1), 6-19. Recuperado en: http://dialnet. unirioja.es/servlet/articulo?codigo $=3122346$

Casado, L. (2001). Organizaciones y sistemas humanos. Una nueva gestión empresarial. Barcelona: Ediciones Kairós.

Cummings T., G. y Worley C., G. (2007). Desarrollo organizacional y cambio. México: Thomson.

Eisler, R. (1998a). El cáliz y la espada. La mujer como fuerza de la historia. Chile: Editorial Cuatro Vientos.

Eisler, R. (1998b). Placer sagrado. Chile: Editorial Cuatro Vientos.

Estebaranz, A. (2000). Construyendo el cambio: perspectivas y propuestas de innovación educativa. España: Secretariado de Publicaciones de la Universidad de Sevilla.

Hellriegel, D. \& Slocum J., W. (2009). Comportamiento organizacional. México: CENGAGE Learning. Lessem, R. (1992). Gestión de la cultura corporativa. Madrid: Ediciones Díaz de Santos, S.A. 
Maturana R., H. (1992). El sentido de lo humano. Chile: Ediciones Pedagógicas Chilenas, S.A.

Maturana R., H. (2007). Transformación en la convivencia. España: Dolmen Ediciones.

Menchén, B. F. (2009). La creatividad y las nuevas tecnologías en las organizaciones modernas. Madrid: Ediciones Díaz de Santos.

Morín, E. (2003). El método V. La humanidad de la humanidad. Madrid: Ediciones Cátedra.

Morin, E. (2006). El método VI. Ética. Madrid: Editorial Cátedra.

Najmanovich, D. (2008). Mirar con nuevos ojos. Nuevos paradigmas en la ciencia y pensamiento complejo. Buenos Aires: Editorial Biblos.

Novo, M. (2007). Mujer y medio ambiente. Los caminos de la visibilidad: Utopías, educación y nuevo paradigma. Madrid: Editorial Catarata.

Pérez L. J., A. (1991). Teoría de la acción humana en las organizaciones. Madrid: Ediciones RIALP, S.A.

Puchol, L. (2005). Nuevos casos en dirección y gestión de recursos humanos. Madrid: Ediciones Díaz de Santos.

Sheldrake, R. (1994). El renacimiento de la naturaleza: La nueva imagen de la ciencia y de Dios. Barcelona: Editorial Paidós.

Swimme, B. (1998). El universo es un dragón verde. Chile: Editorial Cuatro Vientos. 